A time-dependent momentum-space density functional theoretical approach for electron transport dynamics in molecular devices

This content has been downloaded from IOPscience. Please scroll down to see the full text. 2009 EPL 8817008

(http://iopscience.iop.org/0295-5075/88/1/17008)

View the table of contents for this issue, or go to the journal homepage for more

Download details:

IP Address: 129.237.45.148

This content was downloaded on 25/11/2014 at 19:22

Please note that terms and conditions apply. 


\title{
A time-dependent momentum-space density functional theoretical approach for electron transport dynamics in molecular devices
}

\author{
ZhongYuAn ZhOU (a) and SHiH-I CHU \\ Department of Chemistry, University of Kansas - Lawrence, KS 66045, USA
}

received 18 April 2009; accepted in final form 24 September 2009

published online 27 October 2009

PACS 73.63.-b - Electronic transport in nanoscale materials and structures

PACS 85.65.th - Molecular electronic devices

PACS 71.15.Pd - Molecular dynamics calculations (Carr-Parrinello) and other numerical simulations

\begin{abstract}
We propose a time-dependent density functional theoretical (TDDFT) approach in momentum $(\mathcal{P})$ space for the study of electron transport in molecular devices under arbitrary biases. The basic equation of motion, which is a time-dependent integrodifferential equation obtained by Fourier transform of the time-dependent Kohn-Sham equation in spatial coordinate $(\mathcal{R})$ space, is formally exact and includes all the effects and information of the electron transport in the molecular devices. The electron wave function is calculated by solving this equation in a finite $\mathcal{P}$-space volume. This approach is free of self-energy function and memory term related to the electrodes in the $\mathcal{R}$ space and beyond the wide-band limit (WBL). The feasibility and power of the approach are demonstrated by the calculation of current through one-dimensional systems.
\end{abstract}

Copyright (c) EPLA, 2009

Building molecular electronic devices using individual molecules is one of the ultimate goals in nanotechnology [1-6]. A typical molecular device consists of a molecule coupled to two (or more) electrodes [7]. Electrons in the molecular device can go anywhere along the electrodes during the electron transport and electron wave functions may extend to infinity in spatial coordinate $(\mathcal{R})$ space. Thus, accurate calculation of the electron wave functions in $\mathcal{R}$ space is an intractable task for the study of electron transport.

To resolve this problem, one of the widely used methods is to separate the molecule from the electrodes and treat it as an open system. More specifically, the molecular device is partitioned into left $(\mathrm{L})$, central $(\mathrm{C})$, and right ( $\mathrm{R}$ ) zones [8-14]. The $\mathrm{C}$ zone is chosen to include the molecule and some atomic layers of the electrodes so that the Hamiltonian and the electron density of the $\mathrm{L}$ and $\mathrm{R}$ zones (the semi-infinite electrodes) are accurately described by the equilibrium bulk ones before the bias is applied [8]. To calculate the electron wave functions, one separates the $\mathrm{C}$ zone from the $\mathrm{L}$ and $\mathrm{R}$ zones and treat the $\mathrm{C}$ zone as an open system. The effect of the $\mathrm{L}$

\footnotetext{
(a) E-mail: zyzhou@ku.edu
}

and $\mathrm{R}$ zones (environment) on the $\mathrm{C}$ zone (open system) is characterized by a self-energy function [8-16]. After taking this effect into account, an equation of motion for the $\mathrm{C}$ zone is derived. Solving this equation one achieves the electron wave functions for further calculation. This scheme has been extensively applied to the calculation of steady-state current in molecular devices [8-11,17-19].

However, this scheme is not completely applicable when a time-dependent bias is applied [20,21]. In this case, the equation of motion of the $\mathrm{C}$ zone is derived from a timedependent Kohn-Sham (TDKS) equation [12-14]. It not only contains a two-time self-energy function but also a memory term that characterizes the effect of electrodes on the C-zone electrons [12,14-16]. The self-energy function is related to a two-time Green function that can only be readily calculated under the wide-band limit (WBL) [22], an approximation that neglects the energy dependence of the coupling between the molecule and electrodes [15]. Beyond the WBL, the two-time Green function is governed by a double integral equation which is generally difficult to be solved [15]. The memory term related to the electrodes depends on the two-time self-energy function. To calculate this memory term at time $t$, one has to recalculate and store the self-energy function at all the past time steps 
before this time. As a result, computer resources such as CPU time and random-access memory (RAM) required in the calculation become increasingly and thus extremely large with increase of number of time steps.

To overcome these difficulties, a computationally feasible scheme was proposed based on directly solving the TDKS equation [12-14]. In this scheme, the TDKS equation is discretized in the whole $\mathcal{R}$ space and converted to a matrix equation first, then an equation of motion is derived from the matrix equation for the $\mathrm{C}$ zone, and finally the equation of motion is solved to calculate the electron wave functions. This scheme has been successfully applied to the study of one-dimensional (1D) model systems [12-14]. The time-dependent currents achieved tend to the steady-state currents obtained from the Landauer formula $[12,13]$ and Floquet method [14] after a long time, providing a benchmark for the currents through the $1 \mathrm{D}$ model systems. The most striking characteristic of this pioneering scheme is that the effect of $\mathrm{L}$ and $\mathrm{R}$ zones on the $\mathrm{C}$-zone electrons and the reflection of the time-dependent wave function on the boundaries are completely taken good care of by a transparent boundary condition. This scheme can indeed eliminate the explicit dependence of the equation of motion on the two-time self-energy function. However, the equation of motion still contains a memory term used to take into account the effect of electrodes and get rid of the reflection of the electron wave function on the boundaries. As a consequence, the computer resources required in the calculation also increase with the number of time steps.

In fact, the difficulties encountered in the $\mathcal{R}$-space calculations at least come from one of the self-energy function and memory term used to consider the effect of electrodes on the C-zone electrons. Since the electron wave function can extend to the infinity in the molecular device during the transport, the self-energy function or/and memory term related to the electrodes cannot be disregarded no matter how large the $\mathrm{C}$ zone is chosen. Because of this reason, a quantitative study of electron transient transport in the $\mathcal{R}$ space is currently a computational challenge for realistic molecular devices.

However, the momentum of electron is always finite and less than a certain maximum value $\mathbf{k}_{\max }$ in the molecular device. The probability of electron with momentum greater than $\mathbf{k}_{\max }$ is negligible or zero. Thus, in momentum $(\mathcal{P})$ space the electron wave function is localized (e.g., the wave function of a free electron spreads out to the whole space in the $\mathcal{R}$ space but localizes in a very small volume depicted by a $\delta$ function in the $\mathcal{P}$ space) [23] and can be calculated in a finite volume as long as the boundary of the volume is set at the place with a properly large momentum $\mathbf{k}_{\max }$. In this case, all the troublesome terms related to the open system in the $\mathcal{R}$ space, in particular the self-energy function and memory term related to the electrodes, will be wiped out completely and the difficulties encountered in the $\mathcal{R}$-space calculations can be resolved in the $\mathcal{P}$ space. Based on this idea, we propose in this work a first-principles time-dependent density functional theoretical (TDDFT) approach in the $\mathcal{P}$ space for the quantitative study of electron transient transport in realistic molecular devices under arbitrary biases.

The TDDFT approach offers a computationally attractive method for the study of electron dynamics provided an appropriate exchange-correlation (XC) potential functional is employed. For a time-dependent bias the $\mathrm{XC}$ potential may rely on the initial state and history of the system (memory effect) and frequency of the bias. There have been several endeavors to construct such kinds of XC potential functionals for TDDFT approach [24-26]. However, most applications up to date utilize adiabatic approximation where it is assumed that the self-consistent field responds to the applied bias instantaneously and the $\mathrm{XC}$ potential is obtained by plugging in the timedependent density into one of the existing XC potential functionals [27-29]. In the case of low frequency, the validity of the adiabatic approximation was justified by the study of photoionization $[27,30]$. It is shown that neglect of the frequency dependence in the XC potential does not produce significant errors in the calculation of photoionization cross-sections [24]. The applicability of the adiabatic approximation to the high-frequency phenomena was also explored recently through the interaction with strong time-dependent electric fields $[29,31,32]$. It is shown that the memory effect depending on the initial state and history in the $\mathrm{XC}$ potential plays a negligible role in nonlinear and nonperturbative electron dynamics and the adiabatic approximation is rigorously applicable for a finite system of electrons in the high-frequency strong electric field. Therefore the TDDFT approach together with adiabatic XC potentials can be applied to the study of electron transient transport of molecular devices driven by weak and low-frequency time-dependent biases considered here. Nevertheless, in the case of low-frequency limit with constant biases and under stationary conditions, the adiabatic XC potentials used in the TDDFT approach are the same as the self-consistent XC potentials in timeindependent standard density functional theory (DFT). Hence the steady-state currents obtained from the calculation of the TDDFT approach are the same as those from standard DFT-based approaches such as DFT-NEGF approach that combines the DFT with nonequilibrium Green function (NEGF) [8-11,17-19]. However, the standard DFT-based approaches can only apply to the molecular devices with strong coupling between electrodes and molecules [33-36]. Thus the TDDFT approach is justified only for the strongly coupled molecular devices. For weakly coupled molecular devices the results from the calculation of standard DFT-based approaches frequently differ from the experimental data by several orders of magnitude [33,35-38]. These discrepancies are mainly caused by the use of improper XC potentials because in this case many-body correlation effects become large [35] and cannot be characterized by the single-electron XC potentials within DFT. To take these beyond-DFT 
correlation effects into account, an alternative approach has been developed recently based on GW approximation of many-body perturbation theory $[33,35,36,39]$. It is shown that the many-body correlations have large effect on the transient and steady-state currents of molecular devices [34,39] and for the weakly coupled molecular devices the results obtained from this approach are in better agreement with experimental results $[33,36]$.

The molecular device is in an equilibrium state described by a unique temperature and chemical potential when time $t<0$. The charge of the two electrodes is perfectly balanced and no current flows through the device. At time $t=0$ a bias of voltage $v_{b}(t)$ is applied to the electrodes. This bias drives the molecular device out of equilibrium and induces the current through the device.

In the $\mathcal{R}$ space, the time-dependent electron wave function $\psi(\mathbf{r}, t)$ is governed by the TDKS equation within TDDFT approach (atomic units are used throughout the paper)

$$
i \frac{\mathrm{d}}{\mathrm{d} t} \psi(\mathbf{r}, t)=\left[h_{0}(\mathbf{r})+v_{D}(\mathbf{r}, t)\right] \psi(\mathbf{r}, t),
$$

where, $h_{0}(\mathbf{r})$ is the unperturbed KS Hamiltonian of the electron and $v_{D}(\mathbf{r}, t)$ is the driving potential characterizing the effect of bias on the electron. Within the partition above, the unperturbed KS Hamiltonian can be written as $h_{0}(\mathbf{r})=-\nabla^{2} / 2+v_{0}(\mathbf{r})$, where $v_{0}(\mathbf{r})=v_{B K \alpha}(\mathbf{r})$, with $\alpha=L$ and $R$ for the $\mathrm{L}$ and $\mathrm{R}$ zones and $v_{0}(\mathbf{r})=v_{\text {ext }}(\mathbf{r})+$ $v_{\text {eff }}(\mathbf{r}, 0)$ for the $\mathrm{C}$ zone. Here, $v_{B K \alpha}(\mathbf{r})$ is the bulk potential of the electrodes, $v_{\text {ext }}(\mathbf{r})$ is the external potential related to the interaction between the electron and nuclei of the system [40], and $v_{\text {eff }}(\mathbf{r}, 0)$ is the effective potential of the electron comprising the Hartree potential $v_{H}(\mathbf{r}, 0)$ and $\mathrm{XC}$ potential $v_{x c}(\mathbf{r}, 0)$. For the metallic electrodes considered here, the driving potential $v_{D}(\mathbf{r}, t)$ is represented by $v_{L}(t)$ and $v_{R}(t)$ in the $\mathrm{L}$ and $\mathrm{R}$ zones, respectively, and $v_{L}(t)-v_{R}(t)=v_{b}(t)$. These potentials undergo uniform time-dependent shifts due to the screening effect and thus do not change with spatial coordinates if $v_{b}(t)$ is slowly varying during a typical time scale (less than a fs) [13]. In the $\mathrm{C}$ zone, the driving potential $v_{D}(\mathbf{r}, t)$ is represented by $v_{C}(\mathbf{r}, t)$ and $v_{C}(\mathbf{r}, t)=v_{\text {eff }}(\mathbf{r}, t)-v_{\text {eff }}(\mathbf{r}, 0)$ is the perturbing potential induced by the field of bias, where $v_{\text {eff }}(\mathbf{r}, t)$ is the bias-induced effective potential including the Hartree potential $v_{H}(\mathbf{r}, t)$ and $\mathrm{XC}$ potential $v_{x c}(\mathbf{r}, t)$ [30]. The bias-induced effective potential is associated with the response of the $\mathrm{C}$-zone electrons to the field of bias. It may change dramatically and nonlinearly with spatial coordinates [41] and can only be calculated self-consistently with the C-zone electron wave functions. The procedure proposed here is nonperturbative and goes beyond the linear response approximation $[27,30]$. It only applies to the electrons in the $\mathrm{C}$ zone of finite size $[38,42]$.

The relation between the time-dependent $\mathcal{R}$-space electron wave function $\psi(\mathbf{r}, t)$ and $\mathcal{P}$-space electron wave function $\phi(\mathbf{k}, t)$ is given by the Fourier transform $\psi(\mathbf{r}, t)=$ $(2 \pi)^{-3 / 2} \int \mathrm{d} \mathbf{k} \phi(\mathbf{k}, t) \exp (i \mathbf{k} \cdot \mathbf{r})$. Applying this transform to eq. (1), we obtain the $\mathcal{P}$-space TDKS integrodifferential equation

$$
i \frac{\partial}{\partial t} \phi(\mathbf{k}, t)=\int \mathrm{d} \mathbf{k}^{\prime}\left[H_{0}\left(\mathbf{k}, \mathbf{k}^{\prime}\right)+V_{D}\left(\mathbf{k}, \mathbf{k}^{\prime}, t\right)\right] \phi\left(\mathbf{k}^{\prime}, t\right),
$$

where, $H_{0}\left(\mathbf{k}, \mathbf{k}^{\prime}\right)=k^{2} \delta\left(\mathbf{k}-\mathbf{k}^{\prime}\right) / 2+V_{0}\left(\mathbf{k}, \mathbf{k}^{\prime}\right)$ is the $\mathcal{P}$-space unperturbed Hamiltonian, $V_{0}\left(\mathbf{k}, \mathbf{k}^{\prime}\right)$ and $V_{D}\left(\mathbf{k}, \mathbf{k}^{\prime}, t\right)$ are the $\mathcal{P}$-space potentials calculated from the $\mathcal{R}$-space potentials $v_{0}(\mathbf{r})$ and $v_{D}(\mathbf{r}, t)$ by the transform $V_{\alpha}\left(\mathbf{k}, \mathbf{k}^{\prime}\right)=(2 \pi)^{-3} \int \mathrm{d} \mathbf{r} v_{\alpha}(\mathbf{r}) \exp \left[i\left(\mathbf{k}^{\prime}-\mathbf{k}\right) \cdot \mathbf{r}\right]$, with $\alpha=0$ and $D$, respectively.

To calculate the $\mathcal{P}$-space electron wave function, we solve eq. (2) numerically in a finite $3 \mathrm{D}$ volume $\left[-k_{\alpha \max } \leqslant k_{\alpha} \leqslant k_{\alpha \max } \mid \alpha=x, y, z\right]$. The integrals in eq. (2) are discretized using the generalized LegendreGauss-Lobatto pseudospectral method $[31,40,43]$ on $3 \mathrm{D}$ grid points $\left[0,1, \cdots, N_{\alpha} \mid \alpha=x, y, z\right]$ in the finite $3 \mathrm{D}$ volume. Since the $\mathcal{P}$-space electron wave function is localized, the boundary condition for solving eq. (2) is simply that the electron wave function is zero on the boundary. After discretization, eq. (2) is converted to a time-dependent matrix equation

$$
i \frac{\partial}{\partial t} \boldsymbol{\Phi}(t)=\left[\mathbf{H}_{0}+\mathbf{V}_{D}(t)\right] \mathbf{\Phi}(t),
$$

where, $\boldsymbol{\Phi}(t)$ is an $N_{G^{-}}$dimensional vector with the components being the $\mathcal{P}$-space electron wave function $\phi(\mathbf{k}, t)$ on the $3 \mathrm{D}$ grid points, where $N_{G}=N_{x} \times N_{y} \times N_{z}, \mathbf{H}_{0}$ and $\mathbf{V}_{D}(t)$ are $N_{G} \times N_{G}$ matrices with the matrix elements constructed by the unperturbed Hamiltonian $H_{0}\left(\mathbf{k}, \mathbf{k}^{\prime}\right)$ and potential $V_{D}\left(\mathbf{k}, \mathbf{k}^{\prime}, t\right)$ on the $3 \mathrm{D}$ grid points together with the weights of quadrature, respectively.

The $\mathcal{P}$-space electron wave functions at any time can be calculated from eq. (3) if the initial electron wave functions are given. At finite temperature, the electrons populate on the single-electron states of the unperturbed system according to the Fermi distribution function; while in the case of low-temperature limit considered here, the electrons will occupy all the single-electron states from the lowest energy up to the Fermi energy. Thus, the initial electron wave functions are the eigenfunctions of the unperturbed system below the Fermi energy. The eigenvalue equation for the eigenfunctions is obtained by setting $t=0$ in eq. (3) and replacing the term on the left-hand side of eq. (3) with $E \boldsymbol{\Phi}(0)$. Note that $\mathbf{V}_{D}(t)=0$ when $t=0$.

Applying the second-order split-operator method [31] to eq. (3), one obtains the propagation equation $\boldsymbol{\Phi}(t+\Delta t)=$ $\mathbf{P}_{D}(\tau) \mathbf{P}_{0} \mathbf{P}_{D}(\tau) \boldsymbol{\Phi}(t)$, where, $\Delta t$ is the time step size, $\tau=$ $t+\Delta t / 2, \mathbf{P}_{0}=e^{-i \mathbf{H}_{0} \Delta t}$ and $\mathbf{P}_{D}(\tau)=e^{-i \mathbf{V}_{D}(\tau) \Delta t / 2}$ are the propagators, and $\mathbf{V}_{D}(\tau)=\left[\mathbf{V}_{D}(t)+\mathbf{V}_{D}(t+\Delta t)\right] / 2$. The propagators can be calculated using the eigendecomposition scheme of matrix [44]. If the uniform time step size ( $\Delta t=$ constant) is employed, the propagator $\mathbf{P}_{0}$ only needs to be calculated once. In contrast, the time-dependent propagator $\mathbf{P}_{D}(\tau)$ has to be calculated at every time step.

For a finite system the many-body total current is given exactly by the one-electron total current obtained from 


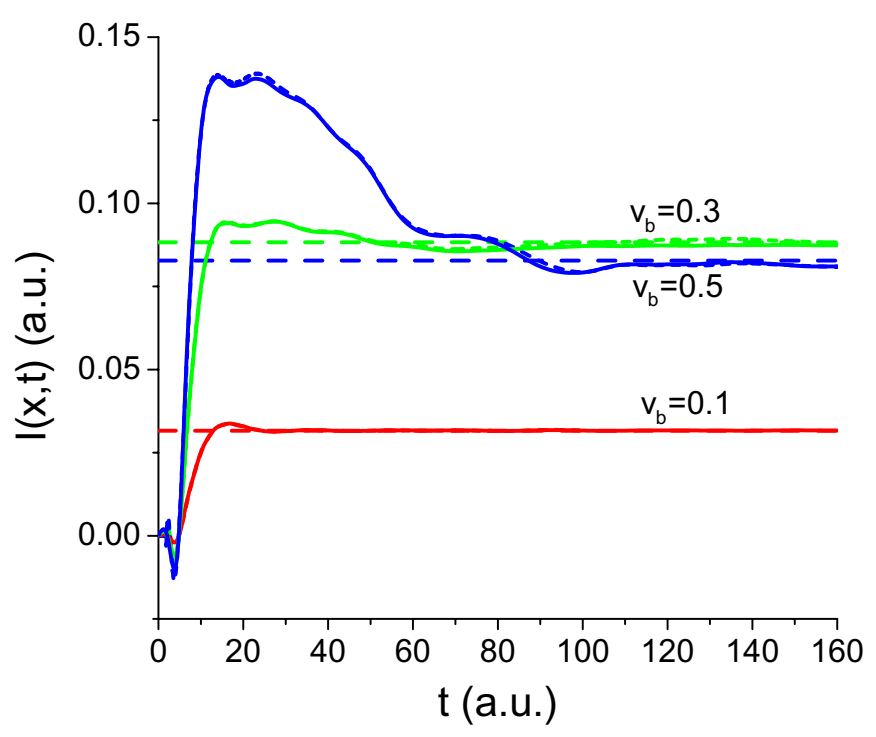

Fig. 1: (Color online) Current densities at $x=0$ for the biases $v_{b}=0.1,0.3$, and 0.5 a.u. For each bias, the shortdashed curve is the result of the $\mathcal{R}$-space transparent-boundary approach $[12,13]$, the dashed line is the steady-state current from the Landauer formula $[12,13]$, and the solid curve is the result of the $\mathcal{P}$-space approach.

TDDFT approach $[41,45,46]$. Using the $\mathcal{P}$-space electron wave functions, the current through the molecular device is calculated by

$$
\begin{aligned}
I(t)= & -\frac{1}{(2 \pi)^{3}} \sum_{j=o c c} \int_{S_{C}} \mathrm{~d} \sigma \widehat{\mathbf{n}} \cdot \operatorname{Re}\left[\int \mathrm{d} \mathbf{k} \phi_{j}^{*}(\mathbf{k}, t) e^{-i \mathbf{k} \cdot \mathbf{r}}\right. \\
& \left.\times \int \mathrm{d} \mathbf{k}^{\prime} \phi_{j}\left(\mathbf{k}^{\prime}, t\right) \mathbf{k}^{\prime} e^{i \mathbf{k}^{\prime} \cdot \mathbf{r}}\right]
\end{aligned}
$$

where, $S_{C}$ is the surface enclosing the $\mathrm{C}$ zone, $\widehat{\mathbf{n}}$ is the unit vector perpendicular to the surface element $\mathrm{d} \sigma, \phi_{j}(\mathbf{k}, t)$ is the $j$-th electron wave function with the momentum $\mathbf{k}$, and the sum $j$ is over all the occupied electron states of the $\mathrm{C}$ zone.

To demonstrate the feasibility of the proposed $\mathcal{P}$-space approach, we first apply it to a $1 \mathrm{D}$ system driven by a DC bias. The potential of the unperturbed $1 \mathrm{D}$ system is zero everywhere in the whole $\mathcal{R}$ space. The $\mathrm{C}$ zone extends from $x=-6$ to +6 a.u. Initially, all the single electron states are occupied up to the Fermi energy $\epsilon_{F}=0.3$ a.u. At $t=0$, a DC bias $v_{b}$ is applied to the electrodes. We choose $v_{L}=-v_{R}=v_{b} / 2, k_{\max }=2.0$ a.u., and $\Delta t=0.1$ a.u. The identical $1 \mathrm{D}$ problem has been considered in refs. $[12,13]$ by using the transparent-boundary approach in the $\mathcal{R}$ space. In fig. 1, we plot the current densities at $x=0$ as a function of time for three biases. It is shown that a steady-state current is achieved for each bias and approaches the steady-state current value obtained from the Landauer formula [12,13]. Furthermore, the currents of the proposed $\mathcal{P}$-space approach are in very excellent agreement with those of the $\mathcal{R}$-space transparent-boundary approach $[12,13]$. However, the computational effort in

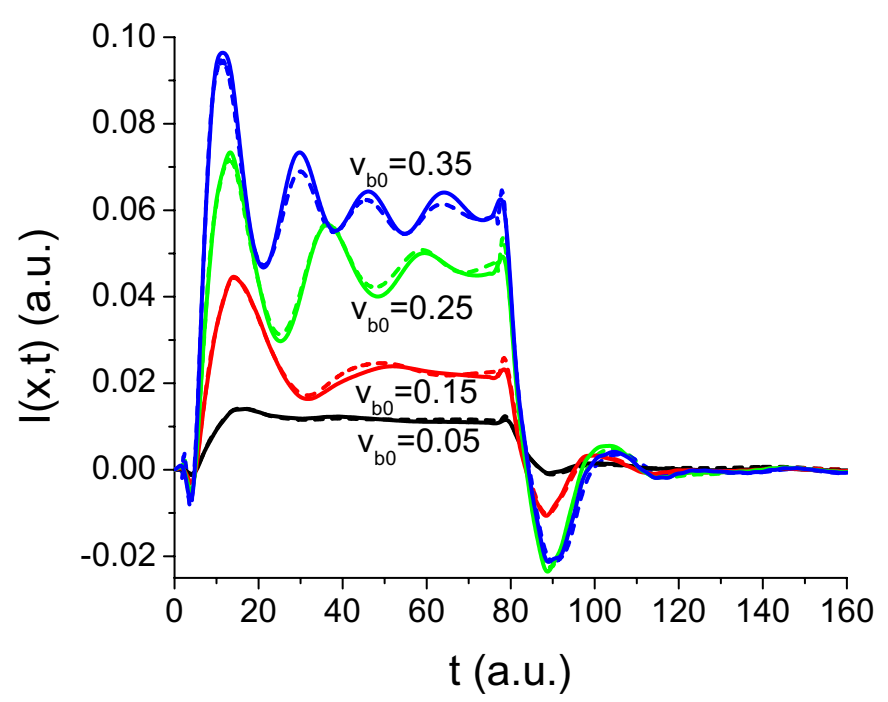

Fig. 2: (Color online) Current densities at $x=0$ for the biases with $v_{b 0}=0.05,0.15,0.25$ and 0.35 a.u. For each bias, the shortdashed curve is the result of the $\mathcal{R}$-space transparent-boundary approach $[12,13]$ and the solid curve is the result of the $\mathcal{P}$-space approach.

the calculation with the $\mathcal{P}$-space approach is considerably smaller than that with the $\mathcal{R}$-space approach.

The second $1 \mathrm{D}$ system we consider is a double square barrier potential driven by a time-dependent bias. The potential of the unperturbed system is $v(x)=0.5 \mathrm{a} . \mathrm{u}$. for $5 \leqslant|x| \leqslant 6$ a.u. and zero otherwise $[12,13]$. The $\mathrm{C}$ zone extends from $x=-6$ to +6 a.u. A time-dependent bias, $v_{b}(t)=v_{b 0}\left[\theta(t)-\theta\left(t-t_{0}\right)\right]$, which is turned on at $t=0$ and turned off at $t=t_{0}(>0)$, is applied to the electrodes, where $\theta$ is the step function and $t_{0}=75 \mathrm{a} . \mathrm{u}$. We choose $\epsilon_{F}=0.3$ a.u., $v_{L}=v_{b}(t), v_{R}=0, k_{\max }=2.0$ a.u., and $\Delta t=$ 0.1 a.u. In fig. 2 we display the current densities at $x=0$ as a function of time for four biases. It is shown that for each bias the current oscillates after the bias is turned off and the amplitude is proportional to the bias. It is also shown that the currents tend to zero steady-state current quickly after turning off the bias and the transient time for turning off a bias is much shorter than that for turning on the bias $[12,13]$. Moreover, the results of the $\mathcal{P}$-space approach are in very good agreement with those of the $\mathcal{R}$-space transparent-boundary approach $[12,13]$. Again the proposed $\mathcal{P}$-space approach reproduces the results of the $\mathcal{R}$-space transparent-boundary approach with considerably less computational effort.

In summary, we propose a first-principles TDDFT approach in the $\mathcal{P}$ space for the study of electron transport dynamics in molecular devices under the arbitrary biases. This approach is effective in theoretical treatment and efficient and feasible in computation. In this approach, the electron wave function is calculated by solving a time-dependent integrodifferential equation in a finite $\mathcal{P}$-space volume. This equation is obtained by the Fourier transform of the $\mathcal{R}$-space TDKS equation. It is exact and contains all the effects and information of the electron 
transport of molecular devices. It is free of the tricky self-energy function and memory term used to account for the effect of electrodes on the C-zone electrons in the $\mathcal{R}$ space and beyond the extensively used WBL. In addition, unlike the $\mathcal{R}$-space approach, the procedure based on this approach does not need to impose any complicated boundary condition to the electron wave functions. The computer resources such as CPU time used at each time step and the RAM required in the calculation with this approach do not increase with the number of time steps. The proposed $\mathcal{P}$-space approach has been successfully applied to the study of 1D systems with less computational effort, demonstrating that it is promising to extend the proposed approach to the study of electron transport dynamics in realistic molecular devices.

ZZ is grateful to Dr G. STEFAnUCCI for helpful discussions on the $\mathcal{R}$-space transparent-boundary approach. This work is partially supported by the Chemical Sciences, Geosciences and Biosciences Division of the Office of Basic Energy Sciences, Office of Science, U. S. Department of Energy, and by the National Science Foundation.

\section{REFERENCES}

11 Collier C. P., Wong E. W., Belohradský M., Raymo F. M., Stoddart J. F., Kuekes P. J., Williams R. S. and Heath J. R., Science, 285 (1999) 391.

[2] Chen J., Reed M. A., Rawlett A. M. and Tour J. M., Science, 286 (1999) 1550.

[3] Rueckes T., Kim K., Joselevich E., Tseng G. Y., Cheung C.-L. and Lieber C. M., Science, 289 (2000) 94.

[4] Tans S. J., Verschueren J., Alwin R. M. and Dekker C., Nature (London), 393 (1998) 49.

[5] Joachim C., Gimzewski J. K. and Aviram A., Nature (London), 408 (2000) 541.

[6] Park J., Pasupathy A. N., Goldsmith J. I., Chang C., Yaish Y., Petta J. R., Rinkoski M., Sethna J. P., Abruna H. D. and McEuen P. L. et al., Nature (London), 417 (2002) 722.

[7] Datta S., Quantum Transport: Atom to Transistor (Cambridge University Press, Cambridge) 2005.

[8] Taylor J., Guo H. and Wang J., Phys. Rev. B, 63 (2001) 245407.

[9] Larade B., Taylor J., Mehrez H. and Guo H., Phys. Rev. B, 64 (2001) 075420.

[10] Brandbyge M., Mozos J. L., Ordejón P., Taylor J. and Stokbro K., Phys. Rev. B, 65 (2002) 165401.

[11] Ke S.-H., Baranger H. U. and Yang W., Phys. Rev. $B, 70$ (2004) 085410.

[12] Kurth S., Stefanucci G., Almbladh C.-O., Rubio A. and Gross E. K. U., Phys. Rev. B, 72 (2005) 035308.

[13] Stefanucci G., Kurth S., Gross E. K. U. and Rubio A., in Molecular and Nano Electronics: Analysis Design and Simulation, edited by SEMinario J. M. (Elsevier) 2006, p. 247.
[14] Stefanucci G., Kurth S., Rubio A. and Gross E. K. U., Phys. Rev. B, 77 (2008) 075339.

[15] Maciejko J., Wang J. and Guo H., Phys. Rev. B, 74 (2006) 085324.

[16] Zheng X., Wang F., Yam C. Y., Mo Y. and Chen G., Phys. Rev. B, 75 (2007) 195127.

[17] Taylor J., Brandbyge M. and Stokbro K., Phys. Rev. $B, 68$ (2003) 121101(R).

[18] Taylor J., Brandbyge M. and Stokbro K., Phys. Rev. Lett., 89 (2002) 138301.

[19] Kaun C.-C., Larade B. and Guo H., Phys. Rev. B, 67 (2003) 121411(R).

[20] Cini M., Phys. Rev. B, 22 (1980) 5887.

[21] Jauho A.-P., Wingreen N. S. and Meir Y., Phys. Rev. $B, 50$ (5528) 1994.

[22] Wingreen N., Jacobsen K. and Wilkins J., Phys. Rev. $B, 40$ (1989) 11834.

[23] Zhou Z. and Chu S.-I., unpublished (2001).

[24] Gross E. K. U. and Kohn W., Phys. Rev. Lett., 55 (1985) 2850.

[25] Vignale G. and Kohn W., Phys. Rev. Lett., 77 (1996) 2037.

[26] Vignale G., Ullrich C. A. and Conti S., Phys. Rev. Lett., 79 (1997) 4878.

[27] Zangwill A. and Soven P., Phys. Rev. A, 21 (1980) 1561.

[28] Ullrich C. A. and Burke K., J. Chem. Phys., 121 (2004) 28.

[29] Thiele M., Gross E. K. U. and Kümmel S., Phys. Rev. Lett., 100 (2008) 153004.

[30] Zhou Z. and Chu S. I., Phys. Rev. A, 79 (2009) 053412.

[31] Chu S. I., J. Chem. Phys., 123 (2005) 062207.

[32] Baer R., J. Mol. Struct.: THEOCHEM, 914 (2009) 19.

[33] Darancet P., Ferretti A., Mayou D. and Olevano V., Phys. Rev. B, 75 (2007) 075102.

[34] Thygesen K. S., Phys. Rev. Lett., 100 (2008) 166804.

[35] Thygesen K. S. and Rubio A., Phys. Rev. B, 77 (2008) 115333.

[36] Wang X., Spataru C. D., Hybertsen M. S. and Millis A. J., Phys. Rev. B, 77 (2008) 045119.

[37] Delaney P. and Greer J. C., Phys. Rev. Lett., 93 (2004) 036805.

[38] Koentopp M., Chang C., Burke K. and Car R., J. Phys.: Condens. Matter, 20 (2008) 083203.

[39] Myöhänen P., Stan A., Stefanucci G. and van LEEUWEN R., EPL, 84 (2008) 67001.

[40] Zhou Z. and Chu S. I., Phys. Rev. A, 71 (2005) 022513.

[41] Sai N., Zwolak M., Vignale G. and Ventra M. D., Phys. Rev. Lett., 94 (2005) 186810.

[42] Galperin M. and Tretiak S., J. Chem. Phys., 128 (2008) 124705.

[43] Canuto C., Hussaini M. Y., Quarteroni A. and ZANG T. A., Spectral Methods in Fluid Dynamics (SpringVerlag, New York) 1988, Chapt. 2.

[44] Strang G., Introduction to Linear Algebra, 3rd edition (Wellesley-Cambridge Press) 1998.

[45] Di Ventra M. and Todorov T. N., J. Phys.: Condens. Matter, 16 (2004) 8025.

[46] Stefanucci G. and Almbladh C.-O., Europhys. Lett., 67 (2004) 14. 